\title{
DIABETES CLASE "R" Y EMBARAZO
}

\section{(Presentación de un caso)}

TRABAJO REALIZADO EN EL SERVICIO DE FISIOLOGIA OBSTETRICA, HOSPITAL DE CLINICAS, UNIVERSIDAD DE LA REPUBLICA, MONTEVIDEO, URUGUAY.

\author{
Dr. Jaime Barrios* \\ Dr. José Rubén López Canales**
}

\section{Introducción}

El interés de la embarazada complicada con diabetes se ha incrementado desde 1930, como resultado de un aumento en la incidencia de embarazo on algunas pacientes. El uso de preparaciones insulínicas de acción lenta en combinación con "insulinas regulares" ha reducido el riesgo de mortalidad materna aproximándose a las no diabéticas con complicaciones vasculares (14). Obviamente, todo este adelanto nos está permitiendo ver los casos más severos de diabetes y de más larga duración combinada con embarazo. El presente trabajo no es sino el reflejo de la era postinsulínica, en donde ya estamos viendo los casos que han obligado a sumar más gravedad de clases a las clasificaciones iniciales de diabetes (9).

\section{Presentación del Caso}

M. E. P., 33 años, soltera, que ingresa al Servicio de Ginecología y Obstetricia del Hospital de Clínicas de Montevideo, el 26 de febrero de 1969, con un embarazo de 24 semanas. Peso corporal 47 kgs. y presión arterial de $120 / 70 \mathrm{~mm}$. de $\mathrm{Hg}$.
Diabética desde hace 26 años con antecedentes de múltiples descompensaciones del tipo acidosis diabética y comas hipoglicémicos. Menarca a los 19 años, ciclos menstruales tipo 30/4, con períodos de amenorrea. Grávida 2 Para I. El primer embarazo cursó con polihidramnios, produciéndose muerte fetal (óbito) a las 34 semanas de embarazo.

Ha sido controlada con insulina N.P.H. y cristalina con dosis que oscilan entre 15 y $80 \mathrm{U}$. El examen de fondo de ojo demostró la presencia de una retinopatía proliferante y la biopsia renal permitió hacer el diagnóstico de una glomérulo esclerosis difuso-nodular con lesiones de aterosclerosis moderada y arteriosclerosis severa. Todo esto ha permitido clasificar la paciente como DIABETICA CLASE " $R$ " (tomando como base la clasificación de la Dra. Priscila White) (9).

En el embarazo actual desarrolló polihidramnios, llegando a tener una altura uterina de 42 $\mathrm{cms}$. a las 34 semanas de gestación, que le imposibilitaba mantenerse en decúbito dorsal, por presentar síndrome supino hipotensivo. Durante su internación los valores de glicemia fueron muy variables, presentando cuadros de hipoglicemia, que hicieron ajustar la terapéutica con insulina de acuerdo a estos valores, recibiendo

* Becario del $2^{\circ}$ Curso Latinoamericano de Biología de la Reproducción, Instructor del Departamento de Obstetricia de la Universidad de Cartagena, Colombia.

** Becario del 20 Curso Latinoamericano de Biología de la Reproducción, procedente de la Universidad Nacional Autónoma de Honduras. 


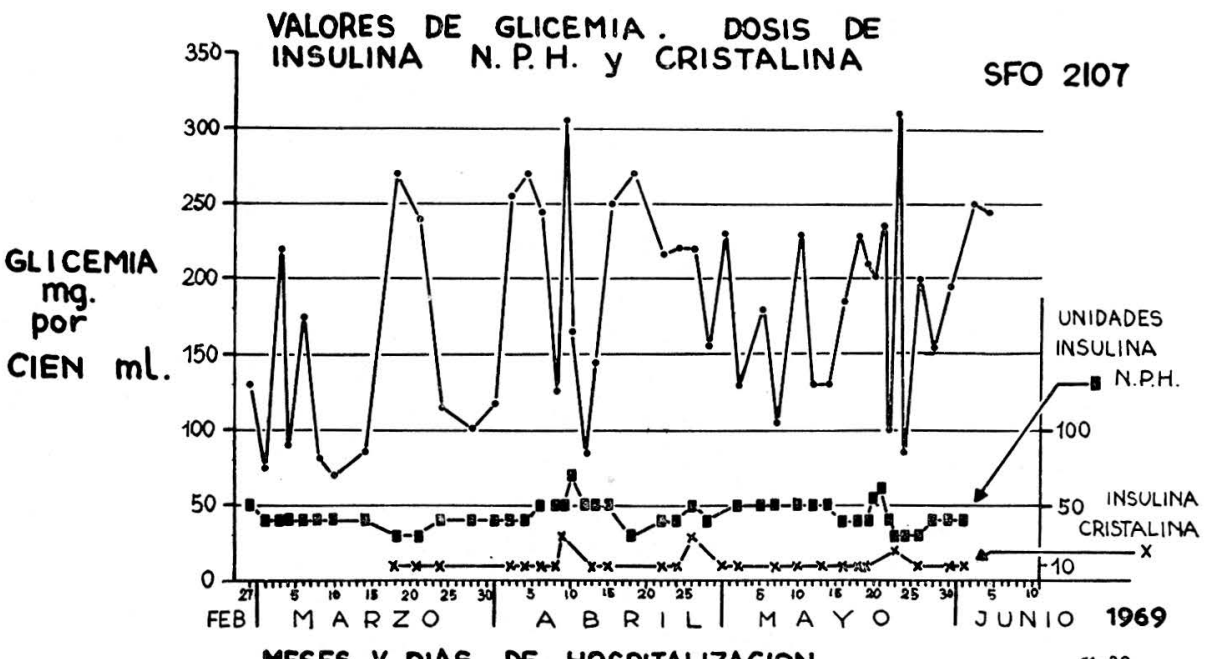

MESES $Y$ DIAS DE HOSPITALIZACION

dosis entre 15 y 80 U.I. de N.P.H. y de 10 a 30 U.I. de insulina cristalina. (Fig. 1).

Los dosajes de estriol urinario a lo largo del embarazo, como se ve en la Fig. 2, se mantuvieron dentro de límites normales, por debajo de la media para la curva de estriol urinario descrita por Brown y col. (con media y D.E.).

Dadas las sospechas de placenta anterior por radiografía, no fue posible hacer punción amniótica para registro de la contractilidad uterina, por lo tanto, para correlacionar los posibles efectos que podría tener las contracciones uterinas inducidas sobre la frecuencia cardíaca fetal (FCF) se hicieron dos pruebas de tolerancia fetal a las contracciones uterinas inducidas con ocitocina sintética (Syntocinon, Sandoz) mediante un registro externo, que consiste en colocar un balón de goma en el abdomen de la madre, fijándolo firmemente. Este balón se conecta mediante un catéter de polietileno a un traductor de presiones y de éste a un polígrafo registrador o Poly-Viso (20).

La frecuencia cardíaca fetal (FCF) se registró colocando en el abdomen un aparato de ultrasonidos (Doptone) que utiliza el efecto doppler (4) (16), y que se integra al polígrafo mediante un cariotacómetro.

En la Fig. 3 registro hecho a las 33 semanas de gestación se observa la FCF en escala de 80 a 180 latidos por minuto (lat $/ \mathrm{min}$.) desde la hora 2:20 a 2:57 del registro. Esta
FCF presentó valores entre 140 y 150 lat $/ \mathrm{min}$. que persistieron durante toda la prueba. En la parte inferior se observa el registro de la contractilidad uterina con $2 \mathrm{mU} / \mathrm{min}$. de ocitocina inyectados a la madre.

Las contracciones con una frecuencia de 4 a 5 en 10 minutos fueron consideradas clínicamente de mediana intensidad ( 25 a $3 \mathrm{~mm}$. Hg.). Este tipo de contractilidad se considera suficiente para evaluar el estado del feto en relación a su sistema cardiovascular (4). Como puede verse en el gráfico, no hubo variaciones marcadas de la FCF durante las contracciones. La ausencia de Dips II (caída de la FSCF Basal) durante las contracciones nos hace pensar que la vitalidad del feto en ese momento es buena y la prueba se considera negativa. Una semana después se le practicó nuevo registro de FCF y contractilidad uterina por método externo resultando también negativa la prueba.

Se le practicó cesárea segmentaria a las 34 semanas y media de embarazo, extrayéndose producto de sexo femenino que pesó $2.000 \mathrm{~g}$. con Apgar de 7 al $1^{\circ}, 5^{\circ}$ y $10^{\circ}$ minutos y que mediante el registro continuo de frecuencia cardíaca del recién nacido y presión arterial se hizo evidente la presencia de taquicardia e hipotensión, sumándose a esto una respiración irregular (polipnea). El estudio seriado de la glicemia de la recién nacida muestra valores en la sangre arterial del cordón umbilical de 494 por $100 \mathrm{ml}$. observándose una progresiva caída a través del tiempo, hasta alcanzar valores de $38 \mathrm{ml}$. por $100 \mathrm{ml}$. a los 2:30 mi- 


\section{GESTA II PARA I EDAD 33 AÑOS \\ DIABETES MELLITUS CLASE "R"}

DOS TESTS DE TOLERANCIA FETAL A LAS CONTRACGIONES UTERINAS NEGATIVOS

(SEMANAS 33 Y 36 ) CESAREA A LAS $361 / 2$ SEMANAS

of PESO: $2.000 \mathrm{~g}$. TALLA $64 \mathrm{~cm}$. APGAR (7) AL 59 MINUTO

\# 2107

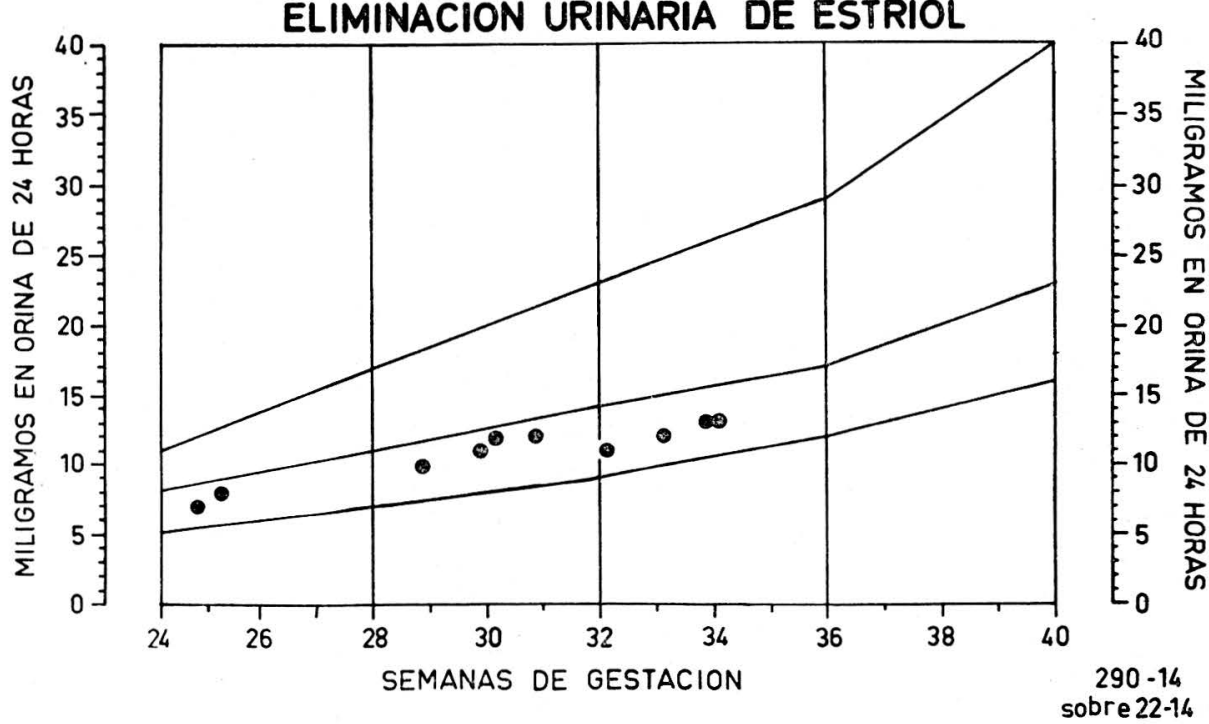

FIGURA $\mathbf{N}^{0} 2$

\#2107 A (PRIMER TEST) REGISTRO EXIERNO

DIABETES MELLITUS CLASE "R" $Y$ POLIHIDRAMNIOS GESTA II EDAD 33 AÑOS

TRATAMIENTO: INSULINA NPH Y CRISTALINA

PRIMER TEST NEGATIYO EN LA SEMANA 33 (DIA 231)

SEGUNDO TEST NEGATIVO EN LA SEMANA 34 (DIA 238)-CESAREA ELECTIVA TRES DIAS DESPUES (DIA 241)

ELIMINACION URINARIA DE ESTRIOL NORMAL DESDE EL DIA 174

BIOPSIA DE LA CADUCA BASAL : ARTERIOLAS NORMALES - BIOPSIA RENAL: GLOMERULO ESCLEROSIS DIFUSO NODULAR RECIEN NACIDO $Q$

PESO: $2.000 \mathrm{~g}$. TALLA: $46 \mathrm{~cm}$

\begin{tabular}{|l|c|c|c|}
\hline APGAR & 7 & 7 & 7 \\
\hline MINUTO & 1 & 5 & 10 \\
\hline
\end{tabular}

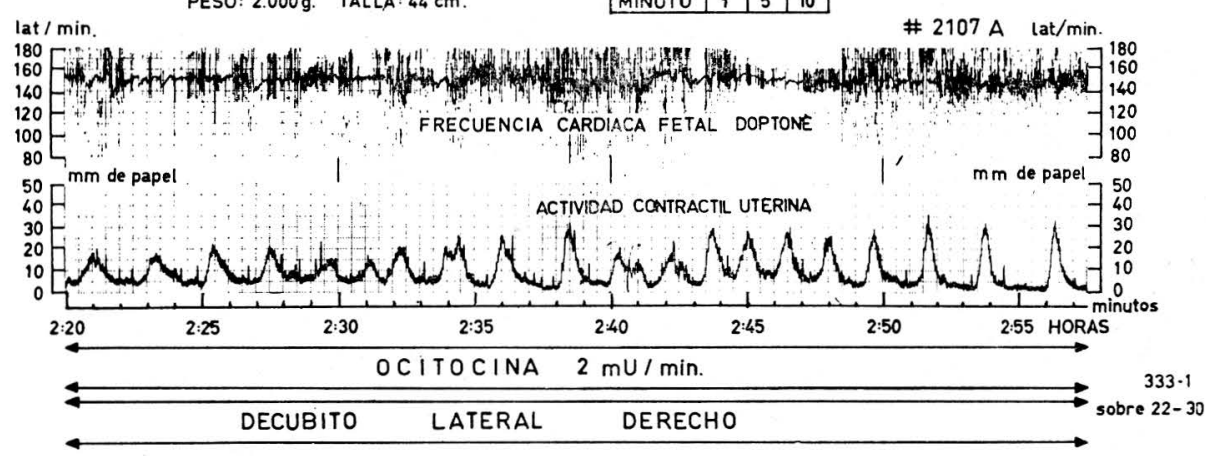

FIGURA $\mathbf{N}^{0} 3$ 


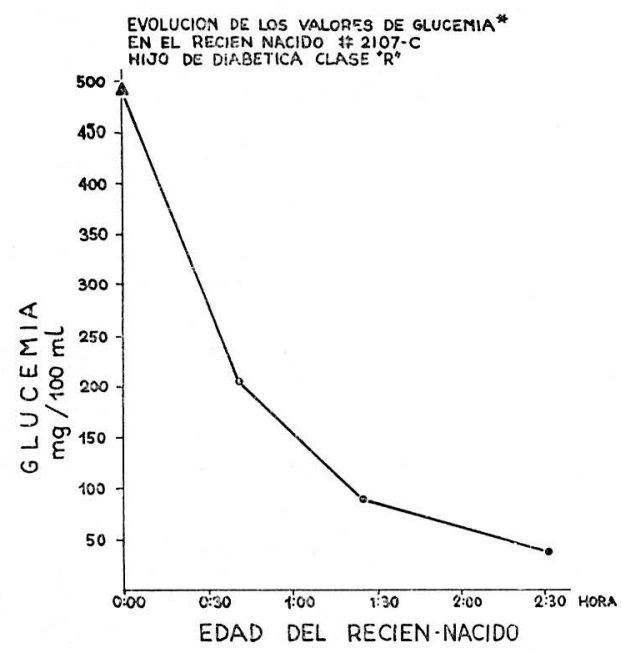

* MicRometodo glUCOSA- OxiDASA

A SANGRe DEL CORDON UMQLLCAL (ARTERIA)

$61-37$
sobre $134-7$

\section{FIGURA $\mathbf{N}^{0} 4$}

nutos de nacida, como puede verse en la Fig. 4.

Fallece a las 18 horas de nacida practicándose la necropsia cuyos hallazgos dignos de destacar son los siguientes: cadáver de prematuro sin malformaciones congénitas; sistema nervioso central: hemorragia intraventricular y hematoma del cuerpo calloso. Pulmones: extensas membranas hialinas interalveolares. Páncreas: ligera hipertrofia de los islotes de langherhans.

En el estudio de la placenta se encontró un sincicio grueso con abundante cantidad de brotes sinciciales $(12,6 \%)$ y abundantes células de Langhans con figuras de mitosis (estudio hecho con microscopía de fase y microscopía óptica). En otros cortes se observan zonas como trombo y hemorragia, nudos sinciciales, dilatación vascular capilar de las vellosidades coriales. Se considera una placenta inmadura.

\section{Discusión}

Bouchardat, Le Corché, Noorden y otros diabetólogos del siglo pasado coincidían en señalar la extraña rareza del embarazo en las diabéticas. Williams, en 1909, en una revisión de literatura mundial hasta esta época, encontró solamente 63 casos de em- barazos en diabéticas, de los cuales algunos eran simples glucosurias. El índice de fertilidad en las mujeres diabéticas era según Le Corché (1885) del 2 al $6 \%$ y no era raro ver cambios regresivos de los órganos genitales y amenorreas secundarias. Las niñas diabéticas morían antes de llegar a la pubertad y las que comenzaban su diabetes en la adolescencia, llegaban difícilmente al matrimonio. La mortalidad fetal era del $50 \%$ y la materna de un $25-50 \%$ (19).

En la era post insulínica la situación precedente cambió. Las niñas que han comenzado su diabetes antes de la pubertad, han podido llegar a la edad adulta, casarse y tener hijos. El número de mujeres diabéticas en condiciones de embarazarse ha ido aumentando por la mayor sobrevida de las pacientes (19).

La enferma presentada ha sido catalogada como diabética clase " $R$ " de la clasificación de Priscina White. austora que ha agrupado esta patología en las siguientes clases (9):

Clase A. "Test de tolerancia a la glucosa diabética", diabetes química, no requiere insulina.

Clase B. Comienzo después de los 20 años, duración menor de e10 años, no enfermedad vascular.

Clase C. Comienzo a los 10-14 años, duración de 10 a 19 años.

Clase D. Comienzo antes de los 10 años, duración mayor de 20 años, hipertensión, retinitis o esclerosis vascular mínima.

Clase F. Nefritis.

Clase " $R$ ". Retinopatía proliferante. 
Ha sido unánimemente aceptado que el polihidramnios es más frecuente en embarazadas diabéticas. Kyle (1963), reportaron una frecuencia de $20 \%$; y posteriormente (1959-1964) $25 \%$. Esta última estadística presenta una frecuencia más alta, porque probablemente contiene más casos severos de la clasificación de P. White. El mecanismo de polihidramnios es aún enigmático (15). Reis y col. han presentado una incidencia de $9,5 \%$ (17), Tillman 9,6\%, Palmer y su grupo $12 \%$, Borns y Morgans $29 \%$ (17), y Carter y col. $33 \%$ (6).

La interrupción de embarazo antes del término ha sido establecida como principio en el manejo de la diabética embarazada, lo cual es un factor importante en la prevención de la muerte intra-útero (9). Sin embargo, la extracción de un feto muy prematuramente puede tener consecuencias graves puesto que, agregada a la alta mortalidad perinatal del hijo de madre diabética, se suma la prematurez y la alta incidencia de membrana hialina.

Si bien la edad cronológica del niño puede representar un mejor índice de madurez, algunos recomiendan que la terminación del embarazo puede ser determinada por estimación del tamaño fetal, aproximadamente 3.500 g. (14). La 35 y 36 semanas son los óptimos períodos para el parto. Si la interrupción se hace antes de la 32 semana, más de la mitad de los recién nacidos morirán de prematurez, y si es después de la 37 semana, más de un cuarto morirán intra-útero (22).

En el caso presentado, dados los antecedentes de polihidramnios $y$ muerte fetal a la semana 34 , se decidió la interrupción de su 2 : embarazo (por cesárea) a la semana 34-
$1 / 2$, edad gestacional en que también apareció polihidramnios.

Wanless ha fijado reglas para indicar el parto por vía vaginal o cesárea. Esta última la decide cuando: hay condiciones desfavorables para la inducción, presentaciones anormales, desproporción feto pélvica, falla de la inducción o no progreso 12 horas después de haber roto membranas, diabetes juvenil en donde el control es difícil, etc. En cambio el parto por vía vaginal lo decide cuando hay un progreso espontáneo, satisfactorio de la labor, condiciones favorables para la inducción, evidencia de anormalidades congénitas fetales y óbito (21).

El test de tolerancia fetal a las contracciones uterinas inducidas, constituye una prueba funcional para explorar la reserva fetal y cuando su resultado es positivo, aparecen dips II, lo que indica que la reserva de oxígeno fetal es baja. Si las contraccioens uterinas no producen dips 11 , el test es negativo $(4,16)$. De acuerdo a la experiencia de ciertos autores, las pacientes clasificadas dentro de las clases más severas ( $P$. White) de enfermedad vascular, tienen menos probabilidad de dar a luz un niño vigoroso. Enfatizan que los resultados obtenidos en diabéticas embarazadas pueden ser correlacionados con la duración de la diabetes y la severidad de la enfermedad vascular (12). Este criterio no es compartido por otros investigadores (14). Ellenberg cree que la duración de la diabetes per se no influencia el pronóstico fetal (7).

El peso del recién nacido del 2 ? embarazo ( 2.000 g.) está por debajo de la media y en el límite del D. E. para la edad gestacional de 34 1/2 semanas de la curva de peso presentada por Hendricks (1968) en recién nacidos normales. En relación a la 
curva de peso de Benedetti y Alvarez, el peso está también por debajo de la media, pero dentro del área correspondiente al D.E. (3).

Se ha difundido el concepto de que los R. N. de madres diabéticas pesan 550 g. más en comparación con niños controles (15). Sin embargo, en reciente series publicadas por Fischer y Moloshok demostraron que casi todos los pesos al nacimiento fueron considerados por encima de los valores normales, pero que, cuando las madres tenían una diabetes de 15 - más años de evolución, los pesos de los niños eran muy semejantes a los de niños de madres normales (10).

El recién nacido de madre diabética tiene una mortalidad alta, evidenciada por un incremento en la frecuencia de membrana hialina. La membrana hialina parece ser el proceso letal más frecuente $v$ es la causa de muerte en el $52 \%$ de las series publicadas por ciertos autores (10). Parece ser que la edad gestacional menor y la diabetes clases avanzadas aumentan la incidencia del "síndrome de dificultad respiratoria" (15).

La más constante observación ha sido la concerniente a la hipertrofia de los islotes de Langherhans del recién nacido de madre diabética; algunos autores como Le Compte y Warren lo han encontrado en un 80$85 \%$ de los casos. Sin embargo, cuantitativos o semicuantitativos estudios son pocos (15).

En relación a la glucemia del recién nacido, el uso de diferentes técnicas para extraer la muestra sanguínea y la aplicación de diferentes métodos de dosaje, ha dificultado la comparación de los resultados de varios investigadores. Farqultar ha mos- trado que la hipoglucemia llega a su más bajo valor a las 2 o 3 horas y qu ees corregida espontáneamente a las 4-6 horas en los hijos de madre diabética (15). Otros han reportado que la glucemia en estos recién nacidos cae más rápidamente durante la primera hora de vida, cae más bajo y retorna a los valores normales lentamente (17). Sin embargo, para otros las curvas de glucemia de hijos de madre diabética son similares a las enocntradas en recién nacidos de madres normale s(1). Pennoyer, Hartman y Reiss, establecieron el nivel más bajo de glucemia de recién nacidos normales en $30 \mathrm{mg} . / 100 \mathrm{ml}$.; en algunos recién nacidos de madres diabéticas se verificaron niveles muy inferiores que llegaron hasta "O" $\mathrm{mg} . / 100 \mathrm{ml}$. (18).

Los valores de estriol urinario están por debajo de la media de la curva descrita por Brow y col. para pacientes normales y coincide con los dosajes encontrados por este autor en embarazadas diabéticas (5). Es opinión casi unánime que no hay muerte fetal cuando la excreción de estriol urinario está por encima de 4 mg./24 hrs. (11). Pedersen y col. han encontrado que en diabéticas embarazadas, los valores de estriol dan bajos, aunque el $80 \%$ de los datos caen dentro del rango normal. Además, en una serie estudiada de diabéticas de la clase $B$ a la $D$ no hubo diferencias de estriol excretado en casos de diabetes ssevera en relación a las menos severas.

Por último, cabe mencionar que aunque muchos autores han reportado innumerables lesiones de la placenta de embarazadas diabéticas, aún no hay una delineación precisa de la patología placentaria en la diabética $(15,17)$. 


\section{Resumen}

Se present aun caso de embarazo y diabetes clase " $R$ " según criterio de clasificación de White. Se discuten varios parámetros estudiados y se relaciona con la experiencia adquirida por otros autores en este campo.

\section{Summary}

A case of pregnancy and diabetes type " $R$ ", according to White's classification, is presented. Several parameters studied are discussed and the patient's history is related to other author's experience in this field.

\section{BIBLIOGRAFIA}

1 YI-YUNG HSIA, D. Direct observations of infants of diabetic mothers. In: Adaptation to Extrauterine Life. Report of the Thirty first Ross Conference on Pediatric Research, 1958, p. 78.

2 APGAR, V. Proposal for new method of evaluation of newborn infant. Anesth \& Analgesic. 32 : 260,1963

3 BENEDETTI, W. y ALVAREZ, H. Evolución del peso fetal y placentario durante el embarazo humano en el Uruguay. Arch. Ginec. y Obst. del Uruguay (En prensa).

4 CASTILLO A., JORGE B. Test de tolerancia fetal a las contracciones uterinas inducidas. Tesis de Doctorad orealizado en el Servicio de Fisiología Obstétrica, de la Facultad de Medicina, Universidad del Uruguay, Montevideo, 1969.

5 COILE, G. MARY and BROW, J. B. Urinary excretion of oestriol during pregnancy. J. Obstet. \& Gynec. Brit. Cmmlth. 70: 224, 1963.

6 CRENSHAW, C., PARKER, ROY T., BAYARD, C. Diabetes mellitus and pregnancy: a 20 year evaluation of 118 pregnancies managed conservatively. Obstet. \& Gynec. 20 : 334, 1962.

7 ELLENBERG, M., RIFKIN, H. Current concepts in Diabetes Mellitus. New York State J. of Medicine. 61: 130, 1961.

8 FRANDSEN, V. A., PEDERSEN, J. STAKEMANN, J. Urinary oestriol excretion in diabetic pregnancy. Acta Endocr., 40: 400, 1962.

9 GARNET, JAMES D. Pregnancy complicated by diabetes. Amer. J. Obstet. \& Gynec. 79 : $140,1960$.
10 GELLIS, SIDNEY S. The newborn infant of the diabetic mother. Clin. Obstet. \& Gynec. 5: 450, 1962.

11 GREENE, JOHN W. Jr., SMITH, K., TOUCHSTONE, JOSEPH C., DUHRING, JOHN L. The use of urinary estriol excretion in the management of pregnancies complicated by Diabetes Mellitus. Amer. J. Obstet. \& Gynec. 91: 684, 1965.

12 CRAMPTEN, JOSEPH, H. Degenerative complication of Diabetes. Clin. Obstet. \& Gynec. 5: 385, 1962.

13 McELIN, THOMAS W. Mode of delivery vaginal or cesarean in Diabetes pregnancies. Cljn. Obstet. \& Gynec. 5: 426, 1962.

14 MCLENDON, HAROLD, BOTTOMY, JOHN R. A critical analysis of the management of pregnancy in diabetic women. Amer. J. Obstet. \& Gynec. 80: 641, 1960.

15 PEDERSEN, JORGEN. The pregnant diabetic and her newborn. Problems and Management. Munksgaard, Copenhagen, Denmark, 1967, pgs. 43, 60, 98.

16 POSE, S. V., CASTILLO, J. B., MORA-ROJAS E. O., SOTO-YANCES, A. Y CALDEYRO-BAR$\mathrm{CIA}, \mathrm{R}$. Prueba de tolerancia fetal a las contracciones uterinas inducidas. Relato oficial del V Congr. Uruguayo de Ginecotocología, Tomo I, Montevideo, Uruguay, 1969. pg. 641.

17 REIS, RALPH, A., DE COSTA, EDWIN J., ALLWEISS, M. DAVID. Diabetes and pregnancy. A monograph in American Lectures in Gynecology and Obstetrics. Editor: E. C. Hamblen, M. D. Publisher: Charles C. Thomas, Springfield, Illinois, USA. 1952. pgs. $31,38,68$.

18 REIS, RALPH, A. Diabetes y embarazo. Conferencia realizada en Montevideo, Uruguay, febrero 1965.

19 ROCCA, F., PLA, J. C. y col. Diabetes MeIlitus. Capítulo XXIV, 1963. Editado por el Departamento de Publicaciones del Sindicato Médico del Uruguay, p. 319.

20 SCHWARCZ, R. L. (h), L., ALTHABE, O. (h). Distocia dinámica (aspectos experimentales). Relato Oficial de la XIV Reunión de la Federación Argentina de Obstetricia y Ginecología. Mendoza, Argentina. 19-23 Septiembre 1966.

21 WANLESS, J. F., Private care in pregnancy complicated by diabetes. Amer. J. Obstet. \& Gynec., 86: 585, 1963.

22 WILSON, R. B. and MORRISON, M. G. Optimal time of delivery of the diabetic patient. Clin. Obstet. \& Gynec., 5: 419, 1962. 